\section{Cryotherapy for retinopathy of prematurity: a MRI study of the surgical- anatomical relationship of the neonatal conjunctival fornices to the globe}

\begin{abstract}
Purpose To demonstrate the surgicalanatomical relationship of the neonatal conjunctival fornices to the globe in order to ascertain whether adequate cryotherapy is possible in infants with stage 3 retinopathy of prematurity using a trans-scleral approach without opening the conjunctiva.

Methods Magnetic resonance (MR) scans of the orbit were performed on an adult and an infant of 38 weeks post-conceptual age with maximal posterior placement of a MRcompatible replica of the Schulenburg cryoprobe in the nasal and temporal aspects of the globe.

Results In the infant MR scan, the scleral indentation produced by the probe was anterior to the equator on the nasal side and at, or just anterior to, the equator on the temporal side. Nasally, the scleral indentation reached only the more anterior part and not the posterior part of zone 2 and none of zone 1 . Temporally, the scleral indentation reached the central part of zone 2 but not the posterior part of zone 2 or zone 1 .

Conclusion The findings suggest that adequate cryotherapy with a trans-scleral approach without opening the conjunctiva is unlikely to be achieved in the more immature neonate with posteriorly located retinopathy of prematurity. To permit access of the cryoprobe to the more posterior part of the globe, one radial conjunctival incision from the corneal limbus in each quadrant may be required to achieve adequate ablation of the posterior non-vascularised retina in these high-risk infants.
\end{abstract}

Key words Conjunctival fornix, Cryotherapy, Neonatal orbit, Retinopathy of prematurity, Scleral indentation
In the United Kingdom, about 6600 infants are born each year with birth weights of under $1500 \mathrm{~g}$. Eighty per cent of these babies survive and approximately $8-10 \%$ of them develop stage 3 retinopathy of prematurity (ROP). ${ }^{1}$ An epidemiological study in the United Kingdom showed an increasing incidence and severity of ROP with increasing immaturity. ${ }^{2,3}$ A baby with gestational age of less than 28 weeks has an $86.7 \%$ chance of developing acute $\mathrm{ROP}^{2}$ and in more immature neonates in whom the retinal vessels have not fully progressed to the periphery, the retinopathy is located more posteriorly. ${ }^{3}$ These babies frequently have extensive areas of non-vascularised retina falling either in zone 1 or more frequently in the posterior part of zone 2. Acute ROP is often more severe on the nasal and temporal sides, with less advanced disease in the upper and lower hemispheres. ${ }^{4}$ To achieve a successful outcome following treatment, an adequate amount of non-vascularised retina needs to be ablated $^{5}$ either by cryotherapy, trans-scleral diode laser, or transpupillary argon or diode laser. Although cryotherapy has been superseded by laser treatment in many units, it remains an important modality of treatment. Argon or diode lasers may not be available in some neonatal units and in some premature neonates transpupillary delivery of lasers may be not be possible due to inadequate media transparency. Radial conjunctival incisions have been used to achieve posterior placement of the cryoprobe.

Parameters of the adult conjunctival fornices and their relation to the corneal limbus and lid margins are well documented. ${ }^{6}$ These data originate from work done in the early part of the twentieth century. However, no data are available on the relationship of the conjunctival fornices to the globe in adults or in neonates. Scleral indentation performed either through
W.E. Schulenburg

Y.F. Yang

E. Parikakis

Western Eye Hospital

London NW1 5YE, UK

\section{G.M. Bydder}

Robert Steiner MR Unit MRC Clinical Sciences

Centre

Hammersmith Hospital London W12 OHS, UK

Mr Yit Fung Yang Moorfields Eye Hospital 162 City Road

London EC1V 2PD, UK

Tel: +44 (0)20 72533411 , pager 144

Fax: +44 (0)20 72534696

e-mail: yyfung@aol.com

Research funding: None

Proprietary interest: None

Received: 20 September 2000 Accepted in revised form: 6 April 2001 


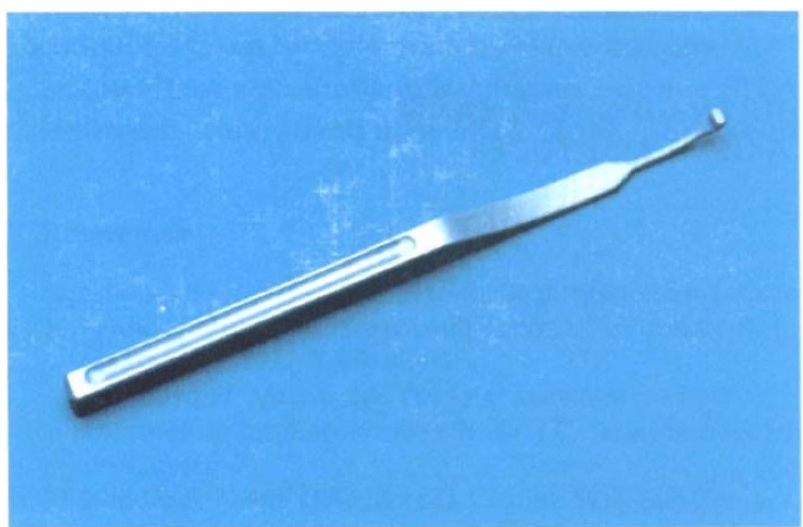

Fig. 1 MR-compatible replica of the Schulenburg cryoprobe.

the palpebral creases or directly through the conjunctival fornices is an important aspect of fundus examination.

An understanding of the anatomical relationship of the conjunctival fornices to the globe in the neonate is essential for carrying out cryotreatment of acute ROP. This study was set up to demonstrate the surgicalanatomical relationship of the conjunctival fornices to the globe in the adult and the neonate using scleral indentation and magnetic resonance (MR) imaging. The aim was to ascertain whether adequate cryotherapy of the posteriorly affected retina in acute ROP, using a trans-scleral approach, is possible without opening the conjunctiva.

\section{Method}

For the purpose of this study, a replica of the Schulenburg cryoprobe made of MR-compatible material (Fig. 1) was prepared. MR imaging (0.5 T, SE 500/20 images of $3 \mathrm{~mm}$ thickness, $192 \times 256$ matrix) employing the replica cryoprobe was first conducted on an adult (W.E.S.). During the scanning process, with the eye in primary position, the replica cryoprobe was placed as posterior as possible in the nasal and temporal conjunctival fornix to the point where diplopia was just noted by the subject. This was to ensure maximal posterior placement of the probe. Gentle pressure was applied on the globe to cause a scleral indentation.

The infant examined in this study was prematurely born at 32 weeks with congenital toxoplasmosis. When examined at 37 weeks post-conceptual age, the right eye was smaller than the left eye and vitreo-retinitis associated with a subtotal retinal detachment was present in the right eye. In the left eye, a vitreous haze was present with a small focal area of retinitis. The left globe was otherwise normal with a corneal diameter of $8.0 \mathrm{~mm}$, which is slightly above the mean corneal diameter for post-conceptional age $(7.79 \mathrm{~mm})$ found in one large study and is well within the $95 \%$ confidence interval for post-conceptional age and birthweight. ${ }^{7}$ The infant had hydrocephalus and cranial toxoplasmosis lesions had to be excluded, which was the clinical indication for the MR scans. Ethics committee approval and the parents' informed consent was obtained for this study.
The MR scan of the left eye was performed under general anaesthesia at the post-conceptual age of 38 weeks. During the scanning process, the replica probe was similarly placed in the nasal and temporal conjunctival fornix to just rotate the globe towards the probe in order to ensure maximal posterior placement of the probe in the conjunctival fornix. Gentle pressure was applied on the globe to cause a scleral indentation. On both the adult and infant MR images, a mark of $7.5 \mathrm{~mm}$ in length representing the radius of zone 1 was included into the perimeter of the globe extending temporally from the centre of the optic disc.

\section{Results}

The MR scan in the adult demonstrated indentation of the sclera at, or just anterior to, the equator on the nasal side (Fig. 2) and just posterior to the equator on the temporal side (Fig. 3). In the infant MR scan with nasal scleral indentation (Fig. 4), the globe was not transected exactly in the mid-horizontal plane. The position of the scleral indentation, however, can be noted to be anterior to the equator of the globe. The scleral indentation was at or just anterior to the equator on the temporal side

(Fig. 5). The posterior limit of the conjunctival fornices is therefore situated slightly more posteriorly in relation to the globe in the adult compared with the neonate. The $7.5 \mathrm{~mm}$ mark demarcating the radius of zone 1 in the scans done in the mid-horizontal plane (Figs. 2, 3 and 5) shows the position of the scleral indent relative to the treatment zones for ROP. In the infant MR scans, nasally, the scleral indent reached only the more anterior part and not the posterior part of zone 2 and none of zone 1. Temporally, the scleral indent can reach the central part of zone 2 but not the posterior part of zone 2 and zone 1 .

\section{Discussion}

Isenberg et al. ${ }^{8}$ published their findings on conjunctival fornix measurements from the lid margin in premature and term infants. Applying their linear regression equations for predicting conjunctival fornix dimensions and using previously published dimensions for the adult and neonatal globe, ${ }^{9-11}$ the ratio of the conjunctival fornix dimensions to the diameter of the globe in the 38 week gestational age infant is much smaller than that of the adult (horizontal dimensions 1.12 vs 1.36 , vertical dimensions 0.93 vs 1.24 ). These calculations seem to suggest that the relationship of the conjunctival fornix to the globe in the premature infant differs significantly from that in the adult, such that scleral indentation without opening the conjunctiva in the premature infant is unlikely to reach as far posteriorly as with adults.

The MR scans performed on the infant of 38 weeks post-conceptual age, showed that the cryoprobe only reached the equator on the temporal side and just anterior to the equator on the nasal side of the neonatal globe because of the restriction due to the depth of the conjunctival fornix. This would correspond with the cryoprobe reaching only the more anterior part but not 


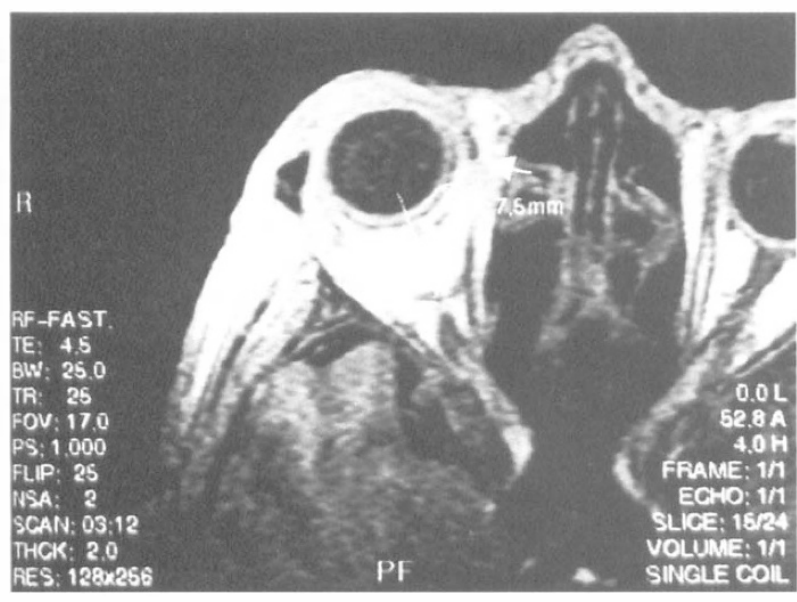

Fig. 2. MR image demonstrating the indentation of the sclera (arrowed) at or just anterior to the equator on the nasal aspect of the adult globe. A $7.5 \mathrm{~mm}$ mark demarcating the radius of zone 1 is included.

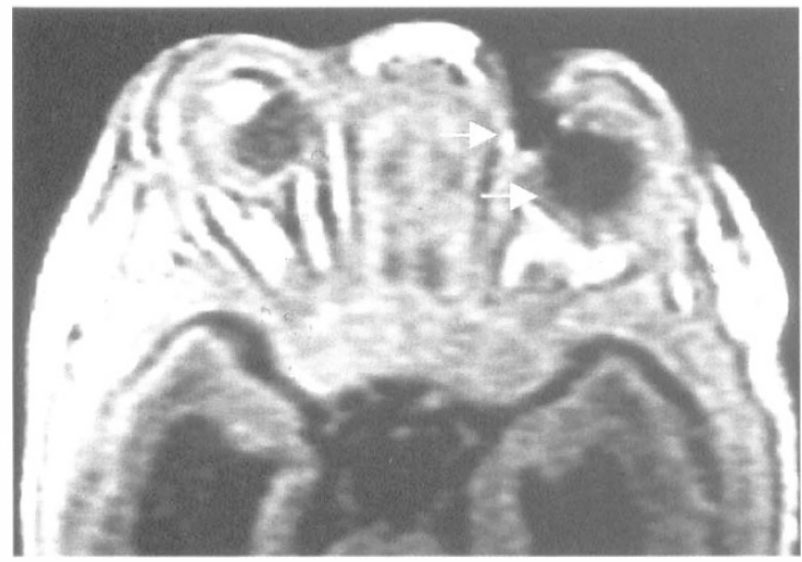

Fig. 4. MR image demonstrating the indentation of the sclera (higher arrow) anterior to the equator on the nasal aspect of the neonatal globe. A second arrow (lower arrow) approximates the location of the equator of the globe.

the posterior part of zone 2 and none of zone 1 nasally. Temporally, the cryoprobe can reach the central part of zone 2 but not the posterior part of zone 2 and zone 1 . The success or failure of treatment of ROP depends on the extent of retinal ablation achieved at the time of treatment. ${ }^{5}$ In more immature infants, in whom the retinal vessels have not progressed so peripherally, threshold ROP may occur with ischaemic nonvascularised retina in zone 1 and frequently in the posterior part of zone 2 which extends well posterior to the equator. ${ }^{3}$ The findings of this study suggest that it is unlikely that adequate retinal ablation would be achieved in these neonates with more posteriorly located retinopathy without opening the conjunctiva. With aseptic techniques, one radial incision in the conjunctiva in each quadrant of the eye allows for cryoprobe to reach the posteriorly placed non-vascularised retina. This also prevents inadvertent tearing of the fragile conjunctiva during treatment. Furthermore, open cryotherapy also decreases the freezing time and unnecessary cryotrauma to the conjunctiva.

The international classification of ROP is based on anatomical landmarks, specifically the optic disc and the ora serrata, other landmarks being difficult to discern in

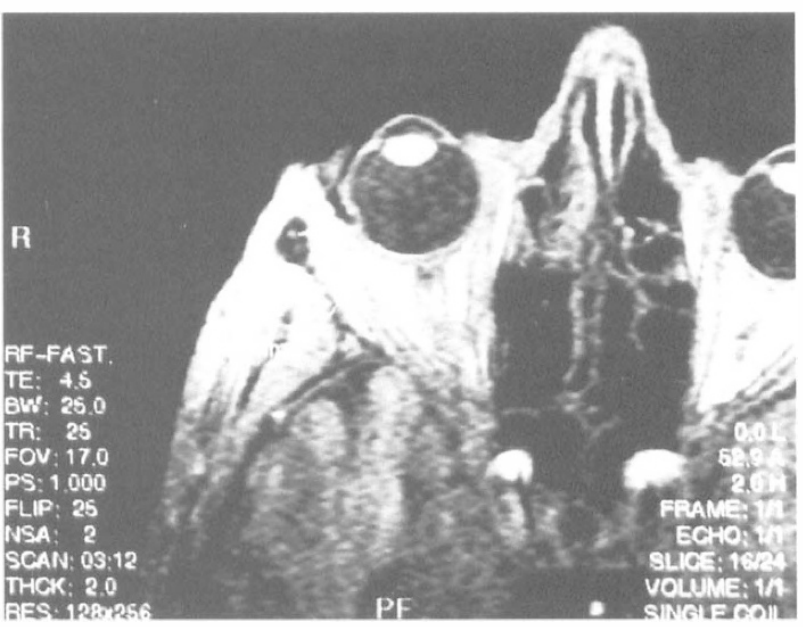

Fig. 3. MR image demonstrating the indentation of the sclera (arrowed) just posterior to the equator on the temporal aspect of the adult globe. A $7.5 \mathrm{~mm}$ mark demarcating the radius of zone 1 is included.

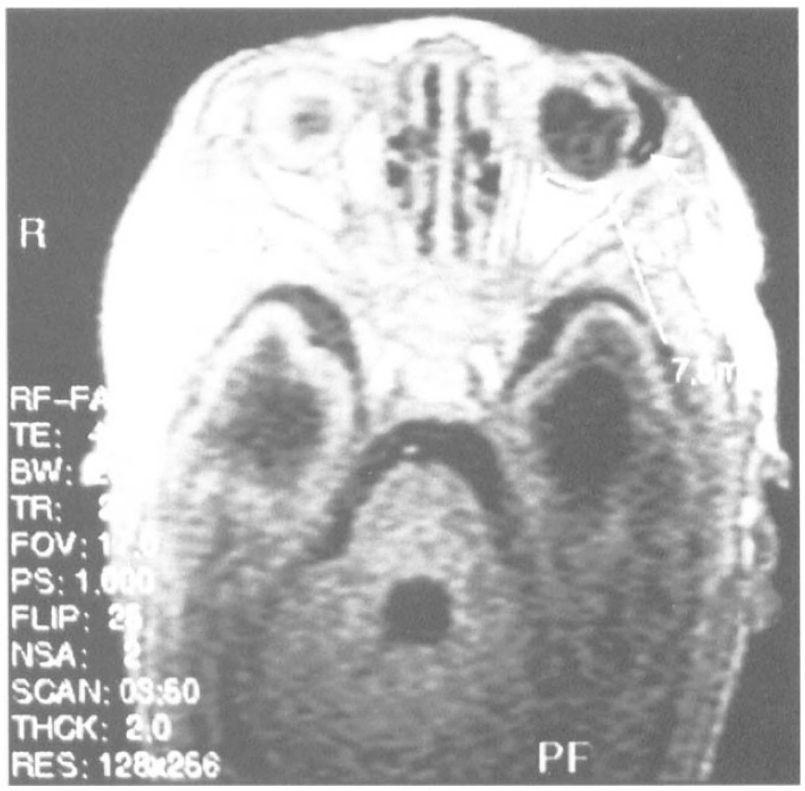

Fig. 5. MR image demonstrating the indentation of the sclera (arrowed) at or just anterior to the equator on the temporal aspect of the neonatal globe. A $7.5 \mathrm{~mm}$ mark demarcating the radius of zone 1 is included.

the premature eye. ${ }^{12}$ Zone 1 extends from the disc to twice the distance from the disc to the centre of the macula, zone 2 extends from the edge of zone 1 peripherally to a point tangenital to the nasal ora and round to an area near the temporal anatomical equator, and zone 3 is the residual temporal crescent of retina anterior to zone 2 . The $7.5 \mathrm{~mm}$ mark on the perimeter of the globe on the MR scan used to demarcate the radius of zone 1 was derived from adult measurements. ${ }^{9}$ Similar dimensions for the premature infant are unavailable and are, conceivably, not amenable to accurate measurement. Previous work by several authors has suggested that the external globe diameter is between 16 and $17 \mathrm{~mm}$ at term, reaching its adult size of $24 \mathrm{~mm}$ during the teens, ${ }^{9-11}$ and that the retinal surface area doubles between 28 weeks of gestation and term and increases another $50 \%$ to reach the adult surface area. ${ }^{13}$ From these figures it would seem very likely that the $7.5 \mathrm{~mm}$ mark 
overestimates the dimensions of zone 1 in the 38 week post-conception infant. This overestimation places the actual border between zone 1 and zone 2 in the premature eye even more posteriorly than indicated by the $7.5 \mathrm{~mm}$ mark in the MR scan, emphasising the discrepancy between the posteriorly located nonvascularised retina which requires ablation and the reach of the cryoprobe from the conjunctival fornices. In the infant MR scan with nasal scleral indentation (Fig. 4), the globe is not transected exactly in the mid-horizontal plane; however, the position of the scleral indent can be noted to be anterior in relation to the approximate position of the equator of the globe. If the mid-horizontal plane view was achieved, the diameter of the globe would appear larger but it would not alter the appearance of the position of the scleral indentation in relation to the equator of the globe. A $7.5 \mathrm{~mm}$ mark was not included in this view (Fig. 4) as it would greatly overestimate the extent of zone 1 in relation to the apparent size of the globe in this scan.

Using MR imaging, this study demonstrated the differences in the relationship between the conjunctival fornix and the globe in the adult and the neonate. The posterior limit of the conjunctival fornices may be situated slightly more posteriorly in relation to the globe in the adult compared with the neonate. More importantly, our findings suggest that, where cryotherapy is used, adequate retinal ablation is unlikely to be achieved without opening the conjunctiva in the more immature neonates in whom the ROP is located more posteriorly. Transpupillary laser may be used to ablate posterior retina if fundus visualisation and mydriasis permit adequate treatment. As only one infant was involved in this study, further studies will be required for a more accurate assessment of the surgicalanatomical relationship of the neonatal conjunctival fornices to the globe.

\section{References}

1. The Royal College of Ophthalmologists, The British Association of Perinatal Medicine. Retinopathy of prematurity: guidelines for screening and treatment. The report of a joint working party, 1995.

2. Ng YK, Shaw DE, Fielder AR, Levene MI. Epidemiology of retinopathy of prematurity. Lancet 1988;I:1235-8.

3. Fielder AR, Foreman N, Moseley MJ, Robinson J. Prematurity and visual development. In: Simons K, editor. Early visual development: normal and abnormal. New York: Oxford University Press, 1993:485-504.

4. Fielder AR, Shaw DE, Robinson J, Ng YK. Natural history of retinopathy of prematurity: a prospective study. Eye 1992;6:233-42.

5. Schulenberg WE, Acheson JF. Cryosurgery for acute retinopathy of prematurity: factors associated with treatment success and failure. Eye 1992;6:215-20.

6. Bron AJ, Tripathi RC, Tripathi BJ. The ocular appendages: eyelids, conjunctiva and lacrimal apparatus. In: Wolff's anatomy of the eye and orbit. 8th ed. London: Chapman and Hall Medical, 1997:30-84.

7. Tucker SM, Enzenauer RW, Levin AV, Morin JD, Hellmann J. Corneal diameter, axial length, and intraocular pressure in premature infants. Ophthalmology 1992;99:1296-300.

8. Isenberg SJ, McCarty JW, Rich R. Growth of the conjunctival fornix and orbital margin in term and premature infants. Ophthalmology 1987;94:1276-80.

9. Bron AJ, Tripathi RC, Tripathi BJ. The eyeball and its dimensions. In: Wolff's anatomy of the eye and orbit. 8th ed. London: Chapman and Hall Medical, 1997:211-32.

10. Weale RA. Biography of the eye: development, growth, age. London: HK Lewis, 1982.

11. Sorsby A, Sheridan. The eye at birth: measurements of the principal diameters in 48 cadavers. Anatomy 1960;94:192.

12. Committee for the classification of retinopathy of prematurity. An international classification of retinopathy of prematurity. Br J Ophthalmol 1984;68:690-7.

13. Robb RM. Increase in retinal surface area during infancy and childhood. J Pediatr Ophthalmol Strabismus 1982;19:16-20. 